Bull. Austral. Math. Soc.

$47 \mathrm{H} 09,54 \mathrm{H} 25$

VOL. 59 (1999) [111-117]

\title{
SOME RESULTS ON COINCIDENCE POINTS
}

\author{
Abdul Latif and Ian Tweddle
}

In this paper we prove some coincidence point theorems for nonself single-valued and multivalued maps satisfying a nonexpansive condition. These extend fixed point theorems for multivalued maps of a number of authors.

\section{INTRODUCTION}

Let $(X, d)$ be a complete metric space, $M$ a nonempty subset of $X$, and for $S=X$ or $S=M$ let $C B(S)$ (respectively $K(S)$ ) denote the family of all nonempty closed bounded (respectively compact) subsets of $S$ endowed with the Hausdorff metric $H$. A multivalued map $T$ of $M$ into $C B(X)$ is called a contraction if there exists a constant $h \in(0,1)$ such that $H(T(x), T(y)) \leqslant h d(x, y)$, for all $x, y \in M$. If we have the Lipschitz constant $h=1$, then $T$ is called a nonexpansive mapping. A point $x$ in $M$ is said to be a fixed point of $T$ if $x \in T(x)$. Nadler [15] and Markin [12] initiated such a geometric approach to multivalued maps. In [15] Nadler proved a fixed point result for multivalued contraction maps of a complete metric space, which is a generalisation of the Banach Contraction Principle. Since then various well-known results for single-valued self contraction and nonexpansive mappings have been extended to multivalued analogues. For example, see $[4,5,9,11,17]$.

On the other hand Kaneko [8] has introduced a notion of multivalued $f$-contraction map as follows. Let $f$ be a single-valued continuous map of $M$ into $X$. Then a multivalued map $T$ of $M$ into $C B(X)$ is called an $f$-contraction if there exists a constant $h \in(0,1)$ such that $H(T(x), T(y)) \leqslant h d(f(x), f(y))$ for all $x, y \in M$. If we have the Lipschitz constant $h=1$, then $T$ is called a $f$-nonexpansive mapping. A point $x$ in $M$ is said to be a coincidence point of $f$ and $T$ if $f(x) \in T(x)$. We denote by $C(f \cap T)$ the set of coincidence points of $f$ and $T$. In [8] Kaneko has proved coincidence and common fixed point results for self $f$-contraction maps, extending results of Jungck [7], Nadler [15] and others. Recently Daffer and Kaneko [2] have studied multivalued $f$-nonexpansive maps and extended results of Smithson [19] and Kaneko [8] for such maps of connected metric spaces, using the concept of an $f$-orbit of the multifunction as a major tool.

Geometric fixed point theory in Functional Analysis for such multivalued maps has been extensively developed. One of its developments has led to substantial weakenings

Received 29th June, 1998

The first author would like to thank the Department of Mathematics, University of Strathclyde, Glasgow and the Commonwealth Scholarship Commission in the UK for their hospitality and support.

Copyright Clearance Centre, Inc. Serial-fee code: 0004-9729/99 \$A2.00+0.00. 
in the assumption that the values of the mapping be subsets of its domain. For example, see $[1,3,6,13,18,20,21,22]$.

In this note we continue the geometric approach and obtain coincidence point results for nonself $f$-contraction and $f$-nonexpansive mappings without commutativity assumptions. In particular, we prove in Section 2 a coincidence point result (Theorem 2.1) for $f$-contraction maps in a complete metrically convex space. At the same time we also obtain a coincidence point result (Theorem 2.2) for such maps satisfying the weakly inward condition in a Banach space, which contains results of Reich [18] and Martinez-Yanez [14] as special cases. Applying these results for $f$-contraction maps in Section 3, we prove some more general results on coincidence points for $f$-nonexpansive maps, which in turn generalise results due to Assad and Kirk [1], Itoh and Takahashi [6], Yanagi [20], Zhang $[22]$, and many others.

First we recall the following definitions. A metric space $X$ is said to be metrically convex [1], if for each $x, y \in M$ with $x \neq y$, there exists $z \in X, x \neq z \neq y$, such that

$$
d(x, z)+d(z, y)=d(x, y) \text {. }
$$

A Banach space $X$ is said to be an Opial space [16] if for each sequence $\left\{x_{n}\right\}$ in $X$ which converges weakly to $x$ and for all $y \neq x$ we have

$$
\liminf _{n \rightarrow \infty}\left\|x_{n} \dot{-} x\right\|<\liminf _{n \rightarrow \infty}\left\|x_{n}-y\right\| .
$$

Hilbert spaces and Banach spaces having weakly continuous duality mappings are Opial spaces $[16]$. On the other hand it is well-known that $L^{p}$ spaces $(p \neq 2)$ are not Opial spaces [9], [16]. A multivalued map $T$ of $M \subseteq X$ into $2^{X}$ (the family of nonempty subsets of $X)$ is said to be: (i) demiclosed if for every sequence $\left\{x_{n}\right\} \subset M$ and any $y_{n} \in T\left(x_{n}\right)$, $n=1,2, \ldots$, such that $x_{n} \stackrel{w}{\longrightarrow} x$ and $y_{n} \rightarrow y$, we have $x \in M$ and $y \in T(x)$. Here and throughout the paper $\rightarrow$ and $\stackrel{w}{\longrightarrow}$ denote strong and weak convergence respectively; (ii) weakly inward if $T(x) \subset \operatorname{cl} I_{M}(x)$ for closed $M$ and $x \in M$, where $I_{M}(x)=\{z \in X$ : $z=x+\lambda(y-x)$ for some $y \in M, \lambda \geqslant 1\}$. The set $I_{M}(x)$ has been called the inward set at $x$.

A subset $M$ is said to be star-shaped with respect to $q \in M$ if $\{(1-\lambda) x+\lambda q: 0<$ $\lambda<1\} \subset M$ for each $x \in M$. The point $q$ is known as a star-centre of $M$. Clearly the star-shaped subsets include the convex subsets as a proper subclass.

\section{CoINCIDENCE POINTS For $f$-CONTRACTION MAPS}

We start with a coincidence point result for complete metrically convex spaces.

THEOREM 2.1. Let $M$ be a nonempty subset of a complete metrically convex space $X$. Let $f: M \rightarrow X$ be any map with its range $G$ closed and $T: M \rightarrow C B(X)$ an $f$-contraction map such that $T(x) \subset G$ for all $f(x) \in \partial G$. Then $C(f \cap T) \neq \emptyset$.

Proof: Define $J: G \rightarrow C B(X)$ by $J(z)=T f^{-1}(z)$ for all $z \in G$. Note that for each $z \in G$ and any $x, y \in f^{-1}(z)$, the $f$-contractiveness of $T$ implies

$$
H(T(x), T(y)) \leqslant h d(f(x), f(y))=0
$$


and hence $J(z)=T(p)$ for all $p \in f^{-1}(z)$. Now we show that $J$ is a contraction. For any $w, z \in G$, we have $H(J(w), J(z))=H(T(x), T(y))$ for any $x \in f^{-1}(w)$ and $y \in f^{-1}(z)$. But $T$ is an $f$-contraction so there exists $h \in(0,1)$ such that

$$
H(J(w), J(z)) \leqslant h d(f(x), f(y))=h d(w, z),
$$

which implies that $J$ is a contraction map. Also note that $J(z) \subset G$ for every $z \in \partial G$. Thus by $\left[1\right.$, Theorem 1], there is a point $z_{0} \in G$ such that $z_{0} \in J\left(z_{0}\right)$. Since $J\left(z_{0}\right)=T\left(x_{0}\right)$ for any $x_{0} \in f^{-1}\left(z_{0}\right)$, so $f\left(x_{0}\right) \in T\left(x_{0}\right)$.

If we take $M$ and $N$ to be subsets of a Banach space, then according to [1] the boundary of a closed set $N$ relative to $M$ is defined by

$$
\partial_{M}(N)=\{a \in N: B(a, r) \cap(M \backslash N) \neq \emptyset \text { for each } r>0\},
$$

where $B(a, r)=\{x \in X:\|x-a\|<r\}$.

Corollary 2.1. Let $M$ be a nonempty closed convex subset of a Banach space, $N$ a subset of $M$. Let $f: N \rightarrow M$ be any map with its range $G$ closed and let $T$ : $N \rightarrow C B(M)$ be an $f$-contraction map such that $T(x) \subset G$ for all $f(x) \in \partial_{M} G$. Then $C(f \cap T) \neq \emptyset$.

Proof: Since in this case $M$ is a complete metrically convex space, the result follows if we replace $M$ by $N$ and $X$ by $M$ in the above theorem.

For a more general boundary condition we have the following coincidence point result for general Banach spaces.

THEOREM 2.2. Let $M$ be a nonempty subset of a Banach space $X$. Let $f$ : $M \rightarrow X$ be any map with its range $G$ closed and $T: M \rightarrow K(X)$ an $f$-contraction map such that $T(x) \subset c l I_{G}(z)$ for all $x \in f^{-1}(z)$. Then $C(f \cap T) \neq \emptyset$.

PROOF: As in the proof of the above theorem, define

$$
J(z)=T f^{-1}(z) \text { for all } z \in G .
$$

Then $J(z)=T(p)$ for all $p \in f^{-1}(z)$ and $J$ is a multivalued contraction map from $G$ into $K(X)$. Also note that $J(z) \subset c l I_{G}(z)$ for any $z \in G$; that is, $J$ is weakly inward. Thus by [21, Theorem 2.1] there exists $z_{0} \in G$ such that $z_{0} \in J\left(z_{0}\right)$ and hence there exists $x_{0} \in M$ such that $f\left(x_{0}\right) \in T\left(x_{0}\right)$.

If $f=I$, the identity on $M$, and $T$ is a single-valued map then we have the following fixed point result of Martinez-Yanez [14].

Corollary 2.2. Let $M$ be a nonempty closed subset of a Banach space $X$. Let $T: M \rightarrow X$ be a weakly inward contraction map. Then $T$ has a unique fixed point.

\section{COINCIDENCE POINTS FOR $f$-NONEXPANSIVE MAPS}

First, for the sake of completeness we give the proof of the following useful lemma $[10]$. 
LEMMA 3.1. Let $M$ be a nonempty weakly compact subset of an Opial space $X$. Let $f: M \rightarrow X$ be a weakly continuous map and $T: M \rightarrow K(X)$ be an $f$-nonexpansive multivalued map. Then $f-T$ is demiclosed.

PROOF: Let $\left\{x_{n}\right\} \subset M$ and $y_{n} \in(f-T) x_{n}$ be such that $x_{n} \stackrel{w}{\longrightarrow} x$ and $y_{n} \rightarrow y$. It is obvious that $x \in M$ and $f\left(x_{n}\right) \stackrel{w}{\longrightarrow} f(x)$. Since $y_{n} \in f\left(x_{n}\right)-T\left(x_{n}\right)$, we get

$$
y_{n}=f\left(x_{n}\right)-u_{n}, \quad \text { for some } u_{n} \in T\left(x_{n}\right) .
$$

Since $T(x)$ is a compact set, there is a $v_{n} \in T(x)$ such that

$$
\left\|u_{n}-v_{n}\right\| \leqslant H\left(T\left(x_{n}\right), T(x)\right) \leqslant\left\|f\left(x_{n}\right)-f(x)\right\| .
$$

From (3.1.1) and (3.1.2), passing to the limit with respect to $n$, we obtain

$$
\liminf _{n \rightarrow \infty}\left\|f\left(x_{n}\right)-f(x)\right\| \geqslant \liminf _{n \rightarrow \infty}\left\|u_{n}-v_{n}\right\|=\liminf _{n \rightarrow \infty}\left\|f\left(x_{n}\right)-y_{n}-v_{n}\right\| .
$$

$T(x)$ being compact, for a convenient subsequence still denoted by $\left\{v_{n}\right\}$, we have $v_{n} \rightarrow$ $v \in T(x)$. Then (3.1.3) yields

$$
\liminf _{n \rightarrow \infty}\left\|f\left(x_{n}\right)-f(x)\right\| \geqslant \liminf _{n \rightarrow \infty}\left\|f\left(x_{n}\right)-y-v\right\| .
$$

Since $X$ is an Opial space and $f\left(x_{n}\right) \stackrel{w}{\longrightarrow} f(x)$, this yields $f(x)=y+v$. Thus $y=$ $f(x)-v \in f(x)-T(x)$, which proves that $f-T$ is demiclosed.

The following result contains Theorem 2 of Assad and Kirk [1], which in turn improved a result of Lami Dozo [9].

TheOREM 3.1. Let $M$ be a nonempty closed convex subset of an Opial space $X$ and $N$ a nonempty weakly compact subset of $M$. Let $f: N \rightarrow M$ be a weakly continuous map with its range $G$ star-shaped and let $T: N \rightarrow K(M)$ be an $f$-nonexpansive map such that $T(x) \subset G$ for $f(x) \in \partial_{M} G$. Then $C(f \cap T) \neq \emptyset$.

PROOF: Let $q$ be a star-centre of $G$; then for any $z \in G$ and any $\lambda(0<\lambda<1)$, $(1-\lambda) z+\lambda q \in G$. Also note that $G$ is closed and bounded. Now, for each $n$, define

$$
T_{n}(x)=\left(1-h_{n}\right) T(x)+h_{n} q
$$

where $x \in N$ and $\left\{h_{n}\right\}$ is any sequence with $h_{n} \rightarrow 0(n \rightarrow \infty)$ and $0<h_{n}<1$. Clearly, for each $n, T_{n}$ maps $N$ into $K(M)$. Now, if $z \in \partial_{M}(G)$, then $T(x) \subset G$ for any $x \in f^{-1}(z)$. Since $G$ is star-shaped with respect to $q$, so $T_{n}(x) \subset G$ for any $x \in f^{-1}(z)$. Furthermore, we have

$$
H\left(T_{n}(x), T_{n}(y)\right) \leqslant\left(1-h_{n}\right)\|f(x)-f(y)\|
$$

for each $n$ and any $x, y \in N$. By Corollary 2.1 there exists $x_{n} \in N$ such that

$$
f\left(x_{n}\right) \in T_{n}\left(x_{n}\right)=\left(1-h_{n}\right) T\left(x_{n}\right)+h_{n} q,
$$

so there is some $u_{n} \in T\left(x_{n}\right)$ such that

$$
f\left(x_{n}\right)=\left(1-h_{n}\right) u_{n}+h_{n} q .
$$


Thus,

$$
\left\|f\left(x_{n}\right)-u_{n}\right\|=\frac{h_{n}}{1-h_{n}}\left\|q-f\left(x_{n}\right)\right\| \rightarrow 0 \text { as } n \rightarrow \infty .
$$

Since $N$ is weakly compact, for a convenient subsequence still denoted by $\left\{x_{n}\right\}$, we have $x_{n} \stackrel{w}{\longrightarrow} x_{0} \in N$. Now as $f\left(x_{n}\right)-u_{n} \in(f-T)\left(x_{n}\right)$ and by Lemma $3.1, f-T$ is demiclosed, we conclude that $0 \in(f-T)\left(x_{0}\right)$ and hence $f\left(x_{0}\right) \in T\left(x_{0}\right)$. This completes the proof.

Applying our Theorem 2.2, we have the following coincidence point results for general Banach spaces.

THEOREM 3.2. Let $M$ be a nonempty subset of a Banach space $X$ and let $f$ : $M \rightarrow X$ with its range $G$ closed, bounded and star-shaped. Let $T: M \rightarrow K(X)$ be an $f$-nonexpansive map which satisfies the following conditions:

(i) $T(x) \subset c l I_{G}(z)$ for all $x \in f^{-1}(z)$

(ii) $(f-T) M$ is closed.

Then $C(f \cap T) \neq \emptyset$.

Proof: Let $q$ be a star-centre of $G$; then $I_{G}(z)$ is also star-shaped with respect to $q$ for each $z \in G$ [22]. For each $n$, define $T_{n}: M \rightarrow K(X)$ by

$$
T_{n}(x)=\left(1-h_{n}\right) T(x)+h_{n} q,
$$

where $x \in M$ and $\left\{h_{n}\right\}$ is any sequence with $h_{n} \rightarrow 0(n \rightarrow \infty)$ and $0<h_{n}<1$. Then it is easy to see that for each $n, T_{n}$ is an $f$-contraction map and $T_{n}(x) \subset \operatorname{clI}_{G}(z)$ for all $x \in f^{-1}(z)$. By Theorem 2.2, there exists $x_{n} \in M$ such that $f\left(x_{n}\right) \in T_{n}\left(x_{n}\right)$ and hence, as in the proof of Theorem 3.1, $f\left(x_{n}\right)-u_{n} \rightarrow 0$ as $n \rightarrow \infty$ for some $u_{n} \in T\left(x_{n}\right)$. Since $(f-T) M$ is closed and $f\left(x_{n}\right)-u_{n} \in(f-T) M$, we get $0 \in(f-T) M$. Hence there is a point $x_{0} \in M$ such that $f\left(x_{0}\right) \in T\left(x_{0}\right)$.

THEOREM 3.3. Let $M$ be a nonempty weakly compact subset of a Banach space $X$ and $f: M \rightarrow X$ a weakly continuous map with its range $G$ star-shaped. Let $T: M \rightarrow$ $K(X)$ be an $f$-nonexpansive map which satisfies the following conditions:

(i) $T(x) \subset c l I_{G}(z)$ for all $x \in f^{-1}(z)$

(ii) $f-T$ is demiclosed.

Then $C(f \cap T) \neq \emptyset$.

Proof: Note that $G$ is weakly compact and hence it is a closed subset of $X$. Let $q$ be a star-centre of $G$; then $I_{G}(z)$ is also star-shaped with respect to $q$. Now, following the proof of the above theorem we get a sequence $\left\{x_{n}\right\}$ in $M$ and $u_{n} \in T\left(x_{n}\right)$ such that $f\left(x_{n}\right)-u_{n} \rightarrow 0$ as $n \rightarrow \infty$. Since $M$ is weakly compact, for a convenient subsequence still denoted by $\left\{x_{n}\right\}$, we have $x_{n} \stackrel{w}{\longrightarrow} x_{0} \in M$. Hence by using demiclosedness of $f-T$, we obtain $0 \in(f-T)\left(x_{0}\right)$, that is, $f\left(x_{0}\right) \in T\left(x_{0}\right)$.

By virtue of Lemma 3.1, we have the following result for Opial spaces.

COROLLARY 3.1. Let $M$ be a nonempty weakly compact subset of an Opial space $X$ and $f: M \rightarrow X$ a weakly continuous map with its range $G$ star-shaped. Let $T: M \rightarrow K(X)$ be an $f$-nonexpansive map such that $T(x) \subset$ clI $_{G}(z)$ for all $x \in f^{-1}(z)$. Then $C(f \cap T) \neq \emptyset$. 
If $f=I$, the identity on $M$, then Theorem 3.3 reduces to the following main fixed point result of Zhang [22], which in turn generalised a result of Yanagi [20].

Corollary 3.2. Let $M$ be a nonempty weakly compact star-shaped subset of a Banach space $X$. Let $T: M \rightarrow K(X)$ be a weakly inward nonexpansive map such that $I-T$ is demiclosed. Then $T$ has a fixed point.

The following result extends a Theorem of Itoh and Takahashi [6].

Corollary 3.3. Let $M$ be a nonempty weakly compact subset of an Opial space $X$ and $f: M \rightarrow X$ a weakly continuous map with its range $G$ star-shaped. Let $T: M \rightarrow K(X)$ be an $f$-nonexpansive map such that for each $z \in \partial G, T(x) \subset$ $G$ for all $x \in f^{-1}(z)$. Then $C(f \cap T) \neq \emptyset$.

Proof: Since for all $z \in G, G \subset I_{G}(z)$ and $I_{G}(z)=X$ if $z$ is an interior point of $G$ [22], thus $T(x) \subset c l I_{G}(z)$ for all $x \in f^{-1}(z)$ and hence the result follows by Corollary 3.1 .

\section{REFERENCES}

[1] N.A. Assad and W.A. Kirk, 'Fixed point theorems for set-valued mappings of contractive type', Pacific J. Math. 43 (1972), 553-562.

[2] P.Z. Daffer and H. Kaneko, 'Multivalued f-contractive mappings', Boll. Un. Mat. Ital. 7 (1994), 233-241.

[3] D. Downing and W.A. Kirk, 'Fixed point theorems for set-valued mappings in metric and Banach spaces', Math. Japon. 22 (1977), 99-112.

[4] K. Goebel, 'On fixed point theorem for multivalued nonexpansive mappings', Ann. Univ. Marie Curie-Sklodowska 29 (1975), 69-72.

[5] T. Husain and A. Latif, 'Fixed points of multivalued nonexpansive maps', Internat. J. Math. Math. Sci. 14 (1991), 421-430.

[6] S. Itoh and W. Takahashi, 'Single-valued mappings, multivalued mappings and fixed point theorems', J. Math. Anal. Appl. 59 (1977), 514-521.

[7] G. Jungck, 'Commuting mappings and fixed points', Amer. Math. Monthly 83 (1976), 261-263.

[8] H. Kaneko, 'Single-valued and multivalued f-contractions', Boll. Un. Mat. Ital. 6 (1985), 29-33.

[9] E. Lami Dozo, 'Multivalued nonexpansive mappings and Opial's condition', Proc. Amer. Math. Soc. 38 (1973), 286-292.

[10] A. Latif and I. Tweddle, 'On multivalued $f$-nonexpansive maps', (submitted).

[11] T.C. Lim, 'A fixed point theorem for multivalued nonexpansive mapping in a uniformly convex Banach space', Bull. Amer. Math. Soc. 80 (1974), 1123-1126.

[12] J.T. Markin, 'A fixed point theorem for set-valued mappings', Bull. Amer. Math. Soc. 74 (1968), 639-640.

[13] S. Massa, 'Some remarks on Opial spaces', Boll. Un. Mat. Ital. 6 (1983), 65-69.

[14] C. Martinez-Yanez, 'A remark on weakly inward contractions', Nonlinear Anal. 16 (1991), 847-848.

[15] S.B. Nadler, 'Multivalued contraction mappings', Pacific J. Math. 30 (1969), 475-488. 
[16] Z. Opial, 'Weak convergence of the sequence of successive approximations for nonexpansive mappings', Bull. Amer. Math. Soc. 73 (1967), 591-597.

[17] S. Reich, 'Fixed points of contractive functions', Boll. Un. Mat. Ital. 4 (1972), 26-42.

[18] S. Reich, 'Approximate selection, best approximation, fixed points and invariant sets', $J$. Math. Anal. Appl. 62 (1978), 104-113.

[19] R.E. Smithson, 'Fixed points for contractive multifunctions', Proc. Amer. Math. Soc. 27 (1971), 192-194.

[20] K. Yanagi, 'On some fixed point theorems for multivalued mappings', Pacific J. Math. 87 (1980), 233-240.

[21] H-W. Yi and Y-C. Zhao, 'Fixed point theorems for weakly inward multivalued mappings and their randomizations', J. Math. Anal. Appl. 183 (1994), 613-619.

[22] S. Zhang, 'Star-shaped sets and fixed points of multivalued mappings', Math. Japon. 36 (1991), 327-334.

Department of Mathematics

Gomal University

Dera Ismail Khan

Pakistan
Department of Mathematics

University of Strathclyde

Glasgow G1 1XH

Scotland

United Kingdom 